\title{
DESEMPENHO DA IMPLEMENTAÇÃO DO PROGRAMA LUZ PARA TODOS: NOVOS ATORES COMO FATOR EXPLICATIVO
}

\author{
'Luz para Todos' Program Implementation Performance
}

\section{RESUMO}

A literatura de implementação de políticas públicas destaca que quanto maior a quantidade de atores envolvidos no processo de execução de determinada política, maiores são as chances de insucesso e de falhas em sua implementação (PRESSMAN; WILDAVSKY, 1984; O'TOOLE; MONTJOY, 1984 e LUNDIN, 2007). Aponta-se, em especial, que há atores não identificados como partes interessadas durante a etapa de elaboração da política que surgem ao longo do processo de implementação de determinada política pública e acabam exercendo influência sobre ela no processo de execução. Neste artigo, busca-se descrever a influência de novos atores no processo de implementação do Programa Luz para Todos e verificar como esse fator se associa à variação de desempenho observada no âmbito da política pública. Os resultados evidenciam que a quantidade de novos atores envolvidos no processo de implementação de uma política pública não é condição necessária e/ou suficiente para explicar o desempenho de sua execução. No entanto, a qualidade da participação dos novos atores se apresenta como fator relevante ao desempenho alcançado.

Palavras-chave: Implementação de Políticas Públicas; Novos atores; Desempenho; Qualitative Comparative Anlysis (QCA); Programa Luz para Todos.

André Dias Oliveira - andredias.eppgg@gmail.com

Mestrando em administração pela UnB.

Artigo submetido no dia 26.11.2012 e aprovado em 18.11.2013

\section{ABSTRACT}

The literature on public policies implementation emphasizes that the larger the amount of actors involved in the execution process of a given policy, larger will be the chances of lack of success and failure in their implementation (PRESSMAN; WILDAVSKY, 1984; O'TOOLE; MONTJOY, 1984 and LUNDIN, 2007). It is particularly stressed that there are non-identified actors as interested parts during the policy elaboration phase, who arise during a given public policy implementation process and end up by exerting some influence on it. The present article intends to describe the influence of new actors in 'Luz para Todos'Program and check how this factor is associated to the performance variation as observed in the scope of this public policy. Results show that the amount of new actors involved in the implementation process of a public policy is not a necessary and/or sufficient condition to explain performance in this public policy execution. However, the new actors participation quality represents a relevant factor in the performance obtained.

Key-words: Implementation of Public Policies; New Actors; Performance; Qualitative Comparative Analysis (QCA); Luz para Todos Program. 


\section{Introdução}

A literatura de implementação de políticas públicas destaca que quanto maior a quantidade de atores que participem do processo de implementação de uma política pública, maiores as chances de insucesso em sua execução. Pressman e Wildavsky (1984) afirmam que o número de atores envolvidos em uma determinada estrutura interorganizacional é um dos fatores que determinam se o processo de implementação de uma política pública será bem-sucedido ou não. Sendo assim, mais atores significam maiores chances de falhas na implantação de determinada política pública. O'Toole e Montjoy (1984) acrescentam que a grande quantidade de atores dificulta o processo de implementação nas situações em que há interdependência recíproca e sequencial entre eles.

Dessa forma, o objetivo desta pesquisa é verificar como a participação de novos atores no processo de implementação do Programa Luz para Todos (Programa Nacional de Universalização do Acesso e Uso da Energia Elétrica) afetou os resultados da execução dessa política pública. ${ }^{1}$ Para os propósitos do trabalho, consideraram-se como novos atores as organizações que não foram identificadas como partes interessadas do Programa LPT durante a fase de elaboração dessa política pública, porém no processo de implementação exerceram influência sobre ela.

Os estudos sobre implementação de políticas públicas estão relacionados ao desenvolvimento de sistemáticas de conhecimento, cujo objetivo é esclarecer como os atores envolvidos nesse processo negociam e conduzem as dificuldades encontradas na execução das políticas públicas (O’Toole, 2000). Dessa forma, compreender a influência da atuação de novos atores no processo de implementação e sua relação com o desempenho dessa política pública pode contribuir para obtenção de subsídios para a literatura de implementação. Importa registrar que este estudo inova ao caracterizar a atuação de novos atores no processo de implementação de uma política pública sob uma ótica diferenciada. A literatura destaca que o número excessivo de novos atores dificulta o processo de implementação (PRESSMAN; WILDAVSKY, 1984; O'TOOLE; MONTJOY, 1984; O'TOOLE, 2000; LUNDIN, 2007). No entanto, a atuação desses novos atores será analisada não do ponto de vista da quantidade dos atores, mas sim da qualidade de sua participação no sentido de facilitar ou atrasar o processo de implementação de uma política pública. Também será analisado o processo de implementação do Programa LPT sob a ótica da influência de novos atores que participaram diretamente do processo de execução dessa política pública.

O Luz para Todos é um programa governamental que tem como objetivo prover acesso à energia elétrica aos domicílios localizados na zona rural. Essa política pública é coordenada pelo Ministério de Minas e Energia (MME), operacionalizado pelas Centrais Elétricas Brasileiras S.A. (Eletrobras) e executado pelas empresas concessionárias e permissionárias de energia elétrica (BRASIL; Ministério de Minas e Energia. Manual de Operacionalização do Programa LPT, 2009), os quais são os atores contemplados na elaboração da política.

Ao longo do processo de implementação do Programa LPT nos respectivos estados brasileiros, surgiram outros atores não identificados na elaboração da política como possíveis interessados em sua execução, 
mas que acabaram exercendo influência direta no processo de implementação.

A seguir, apresenta-se uma breve descrição do Programa Luz para Todos. Após essa contextualização, será discutido 0 marco referencial que orientou o estudo: implementação de políticas públicas; modelos de análise do processo de implementação de políticas públicas; conceito de desempenho de uma política pública, e atuação de novos atores no processo de implementação. Em sequência, haverá caracterização dos métodos utilizados para coletar e analisar os dados, seguida da descrição dos casos, ou seja, a identificação da ausência ou presença de novos atores que participaram do processo de implementação do Programa LPT nos diferentes estados brasileiros e a discussão dos resultados alcançados. Finalizando, apresentam-se as considerações finais.

\section{Programa Luz para Todos}

De acordo com dados levantados pelo Censo de 2000 do Instituto Brasileiro de Geografia e Estatística (IBGE), existiam, no Brasil, naquela época, aproximadamente dois milhões de domicílios rurais, onde moravam em torno de 10 milhões de pessoas, sem acesso à energia elétrica. Verificou-se ainda que as famílias desassistidas desse serviço público estavam majoritariamente situadas nas regiões de menor Índice de Desenvolvimento Humano (IDH) e cerca de $90 \%$ delas possuíam renda inferior a três salários mínimos.

Tendo em vista esse contexto, o Programa Luz para Todos (LPT) - Programa Nacional de Universalização do Acesso e Uso da Energia Elétrica - surgiu como alternativa e estratégia para combater a pobreza e a desigualdade social nessas localidades, tendo em vista que a energia poderia ser considerada como vetor de desenvolvimento socioeconômico (BRASIL; Ministério de Minas e Energia, 2010).

O LPT é um programa governamental criado em 2003, durante o Governo Lula, cujo objetivo é prover acesso à energia elétrica aos domicílios localizados na zona rural (BRASIL; Decreto n. 4.873, de 11 de novembro de 2003). Essa política pública é coordenada pelo Ministério de Minas e Energia (MME), operacionalizado pelas Centrais Elétricas Brasileiras S.A. (Eletrobras) e executado pelas empresas concessionárias e permissionárias de energia elétrica.

Os principais agentes responsáveis pela condução desse programa governamental são: Ministério de Minas e Energia (MME), Agência Nacional de Energia Elétrica (ANEEL), Centrais Elétricas Brasileiras S.A. (Eletrobras); concessionárias e permissionárias de distribuição de energia elétrica; cooperativas de eletrificação rural, e governos estaduais. No entanto, durante - processo de implementação dessa política pública, surgiram outros atores que passaram a exercer influência sobre a execução dessa política, como órgãos de controle ambiental e outras organizações governamentais.

Em relação ao desempenho do Programa LPT durante o período de 2004 a 2008, pode-se destacar que, a respeito do número de ligações realizadas no país, foi satisfatório, tendo em vista que alcançou a marca de $96 \%$ das ligações planejadas. No entanto, a variação de desempenho do LPT entre os estados brasileiros foi muito grande, sendo o melhor no estado do Espírito Santo - $371 \%$, enquanto o pior foi o dos estados 
do Amazonas, Amapá e Piauí - 29\%. Isso significa que houve dificuldade de execução do Programa em algumas localidades e em outras não, a qual pode estar relacionada a diversos fatores, inclusive a atuação de novos atores no processo de implementação dessa política pública.

Observa-se que a execução desse programa é bastante complexa, tendo em vista a quantidade de atores envolvidos no processo de sua implementação e na estrutura de gestão dessa política pública. Além dos atores que estão identificados na estrutura de gestão do LPT, com atribuições bem definidas, há outros que surgiram durante o processo de implementação e interferiram, de forma positiva ou negativa, na execução dessa política pública, sendo relevante investigar seu papel no processo de implementação e sua contribuição ao desempenho verificado nos distintos estados brasileiros.

\section{Marco teórico}

$\mathrm{Na}$ visão clássica da ação governamental, a implementação constitui uma das fases do ciclo de políticas públicas (LOWI, 1972), a qual corresponde à execução de ações implementadas com vistas à obtenção de metas definidas no processo de formulação das políticas (SILVA; MELO, 2000).

Para Howlett e Ramesh (1995), implementação é um processo no qual os programas e as políticas públicas são conduzidos, colocando o que foi planejado em prática. Para Rua (1997), a implementação de políticas públicas pode ser compreendida como o conjunto de ações realizadas por grupos ou indivíduos de natureza pública ou privada, as quais são direcionadas para a consecução de objetivos estabelecidos mediante decisões anteriores.

Pressman e Wildavsky (1984), por sua vez, definem implementação de políticas públicas como o processo de interação entre o estabelecimento dos objetivos e as ações empreendidas para alcançá-los. Para esses dois autores, o processo de implementação está relacionado à habilidade de atingir os objetivos estabelecidos para determinada política pública (PRESSMAN; WILDAVSKY, 1984).

De acordo com Subirats (1992), a análise do processo de implementação de políticas públicas envolve o estudo sistemático das atividades de implementação, cujo objetivo é individualizar os fatores que explicam o processo de transformação dos programas em resultados.

A literatura de implementação de política pública destaca duas vertentes de análise do processo de implementação:

1. Top-down e bottom-up: ressalta que as ações de implementação de uma política pública coincidem com as decisões estabelecidas pelas autoridades que a formularam (MATLAND, 1995). Aborda-se o estudo da etapa de implementação de políticas públicas a partir de uma visão macro, ou seja, daqueles que são os formuladores das políticas.

2. Bottom-up: versa sobre o processo de implementação a partir das perspectivas e expectativas dos beneficiários da política pública e da localidade de sua implantação (MATLAND, 1995). Possui visão mais micro, específica da localidade e dos atores que atuam nesse local.

Conforme Matland (1995), os teóricos da 
vertente top-down enxergam os formuladores de políticas públicas como os atores centrais do processo de sua implementação e concentram sua preocupação nos fatores que podem afetar as decisões no nível central. Os teóricos da vertente bottom-up focam nos beneficiários das políticas públicas e nos agentes implementadores, argumentando que a política pública implementada se define no nível local (MATLAND, 1995).

Observa-se que as abordagens sobre o estudo do processo de implementação de uma política pública se relacionam diretamente à definição de desempenho e sucesso de determinada política (PRESSMAN; WILDAVSKY, 1984; MATLAND, 1995; LUNDIN, 2007). Na visão top-down, o desempenho de implementação de políticas públicas está relacionado ao alcance das metas estabelecidas por seus elaboradores, ou seja, o sucesso na execução de um programa refere-se à aderência ao planejamento, aos objetivos inicialmente definidos e a seu cumprimento (MATLAND, 1995). Em contraposição, os teóricos defensores do modelo bottom-up pregam que o sucesso na implementação de uma política pública não depende apenas do desenho da política elaborado pelos formuladores, mas, principalmente, da adaptabilidade da política pública e da capacidade dos implementadores de torná-la exequível à realidade encontrada (MATLAND, 1995).

Nesse sentido, os teóricos das vertentes topdown e bottom-up divergem quanto à definição de sucesso no processo de implementação. De acordo com os top-downers, o sucesso da implementação está relacionado ao alcance dos objetivos que foram traçados durante a fase de formulação da política. Os bottom-upers acreditam que o sucesso está relacionado a um conceito mais amplo, defendendo que ele esteja intrinsecamente vinculado ao alcance de resultados positivos da política, ou seja, ao impacto (MATLAND, 1995).

As definições de sucesso e insucesso estão diretamente relacionadas ao desempenho de determinada política pública. De acordo com Pressman e Wildavsky (1984) e Lundin (2007), em uma postura que pode ser caracterizada como top-down, desempenho é grau de cumprimento dos objetivos alcançados pela política pública, posição adotada neste artigo.

De acordo com Matland (1995), o grau de ambiguidade inerente a uma política afeta diretamente o processo de implementação. Quando os objetivos da política são claramente explicitados, os comandos dos formuladores possuem um valor maior. Nesses casos, o critério de sucesso seria - alcance dos objetivos expressos na formulação. Quando os objetivos da política não são claramente definidos, a avaliação de sucesso se torna mais difícil, pois diferentes critérios podem ser incluídos na avaliação. As diretrizes das políticas públicas são, com frequência, excessivamente vagas. Elas não incorporam objetivos específicos e falham por não estabelecer marcos para a medição dos resultados. Isso abre um leque bastante extenso de possíveis mensurações (MATLAND, 1995).

Pressman e Wildavsky (1984) sugerem que o desempenho de uma política pública varia de acordo com o número de atores envolvidos no processo de implementação de uma estrutura interorganizacional e afirmam categoricamente que esse aspecto é um dos fatores que determinam se o processo de implementação de uma política pública será bem-sucedido ou não. Sendo assim, 
mais atores significam maiores chances de falhas na implantação de uma determinada política pública. Por sua parte, O'Toole e Montjoy (1984) acrescentam que grande quantidade de atores dificulta 0 processo de implementação nas situações em que há interdependência recíproca e sequencial entre eles. Lundin (2007) destaca também que o desempenho de uma política pública pode ser afetado pela quantidade de atores envolvidos no processo de implementação, enfatizando que a quantidade maior de atores aumentará ainda mais as dificuldades se as tarefas de execução dessa política pública forem complexas.

De acordo com Mazmanian e Sabatier (1989), há uma grande dificuldade para se obter coordenação entre os diversos atores que participam do processo de implementação de uma política pública. O problema aumenta em casos de estados federativos, em que as políticas públicas são elaboradas pelo governo central e implementadas nas localidades. Para contornar esse problema de coordenação, Mazmanian e Sabatier (1989) defendem que haja integração hierárquica entre os atores que participam do processo de implementação e redução do poder de veto deles, o que contribui para que haja menos atrasos e impedimentos na condução de uma política pública.

No estudo realizado por Pressman e Wildavsky (1984), busca-se entender as razões de haver tanta morosidade e dificuldades para a implantação de programas de emprego e redução da pobreza na cidade de Oakland, localizada no estado da Califórnia (EUA), já que a princípio todos os atores envolvidos concordavam com seus pressupostos. Eles concluem que havia uma quantidade enorme de atores envolvidos, inclusive novos atores que inicialmente não foram consultados na ocasião da formulação da política, porém demonstraram interesse direto na implementação do Projeto Oakland. Esses novos atores possuíam expectativas e motivações distintas em relação ao projeto (PRESSMAN; WILDAVSKY, 1984), que ficaram conhecidas somente na fase de execução, afetando negativamente 0 desempenho do processo de implementação dessa política pública.

A literatura deixa claro os novos atores que surgem no processo de implementação das políticas públicas podem ser considerados como um fator que influencia diretamente o desempenho da execução dessa política.

\section{Método}

Examinou-se o processo de implementação do Programa LPT nos estados brasileiros durante o período de 2004 a 2008, com o objetivo de identificar, descrever e caracterizar a influência da atuação de novos atores em seu processo de implementação e como esse fator se associa à variação de desempenho observada no contexto dessa política.

Tendo em vista que há um monitoramento/ acompanhamento sistemático e extensivo do objeto de pesquisa, a dimensão temporal desse estudo é caracterizada como seccional de perspectiva longitudinal (PETTIGREW, 1990). Importa destacar que a primeira fase do Programa LPT (2004-2008) foi escolhida como objeto de estudo deste artigo pelo fato de já possuir informações consolidadas sobre o desempenho dessa política pública nos diferentes estados, o que facilita a análise do fenômeno.

A pesquisa desenvolvida apresenta caráter 
descritivo. Averiguou-se a influência da atuação de novos atores no processo de implementação do Programa LPT e como esse fator se associa à variação de desempenho desse programa nos diferentes estados. A inclusão de apenas "novos atores" na pesquisa se deve a vários fatores. Primeiro, está relacionada a uma estratégia de delimitação do escopo do estudo. Segundo, refere-se à questão de que os atores que foram mapeados previamente no processo de formulação da política pública possuem suas atribuições claramente delimitadas e suas ações estão mais coordenadas e articuladas. Por último, os "novos atores" são elementos "surpresa" no processo de implementação, razão pela qual se focou na atuação desses novos atores que influenciaram diretamente a execução dessa política pública.

A abordagem metodológica da pesquisa é qualitativa. No entanto, foram utilizados dados qualitativos e quantitativos para a realização das análises. O uso de diferentes tipos de dados é importante para complementar lacunas de informação que possam existir durante a concretização do estudo (CRESWELL, 2010). A metodologia qualitativa constitui-se de técnicas de pesquisa documental e entrevistas semiestruturadas. Foram encaminhados questionários para os integrantes de todos os Comitês Gestores Estaduais do Programa LPT, os quais foram utilizados para realizar a triangulação com os dados qualitativos da pesquisa, objetivando fortalecer a análise (SCHWARTZ-SHEA, 2006).

O propósito deste estudo é descrevera influência da atuação de novos atores no processo de implementação do Programa LPT e como ela se associa à variação de desempenho, observada no contexto dessa política pública nos diferentes estados brasileiros. Para tanto, foi escolhida a estratégia de múltiplos casos
- a implementação do Programa LPT nos diferentes estados brasileiros - para a realização desta pesquisa. Essa estratégia se caracteriza pelo "[...] exame detalhado de um aspecto de um episódio histórico com o intuito de desenvolver ou testar explicações que podem ser generalizadas para outros eventos" (GEORGE; BENNET, 2005, tradução livre).

Após a realização dos estudos de múltiplos casos - a implementação do Programa LPT em cada estado brasileiro foi tratada como um caso -, foi aplicado o método de análise comparativa, que envolve a investigação de diversas unidades de análise com o intuito de ressaltar diferenças e similaridades entre os diferentes casos estudados (RAGIN, 1987). Utiliza-se o método comparativo para compreender fenômenos complexos, pois possibilita identificar e descrever situações em que diferentes arranjos de variáveis independentes podem conduzir a um mesmo resultado em casos distintos (GEORGE; BENNET, 2005). Nesse sentido, a análise comparativa permite identificar a complexidade causal por meio da identificação de condições necessárias e/ou suficientes para um dado resultado. Sendo assim, possibilita distinguir os conjuntos ou combinações de fatores que caracterizam os diferentes desempenhos alcançados pelos estados brasileiros durante o processo de implementação do Programa LPT, sob a ótica da atuação ou não de novos atores nesse processo.

As perguntas norteadoras deste artigo são: "Como se caracteriza a atuação de novos atores no processo de implementação do Programa LPT e qual o resultado da influência de suas ações no desempenho desse processo nos diferentes estados 
brasileiros?".

Em relação às variáveis que fazem parte da pesquisa maior que deu origem a este artigo, destacam-se a dependente/consequente (Desempenho do Programa Luz para Todos) e a independente/antecedente (Novos atores).

A variável dependente/consequente (Desempenho do Programa Luz para Todos) foi operacionalizada em razão da quantidade de ligações de energia elétrica efetuadas pelo Programa LPT nos diferentes estados brasileiros, em comparação com as metas de ligações estabelecidas pelo programa para cada estado. Sendo assim, a variável "Desempenho" está relacionada ao que, de fato, o Programa LPT realizou em comparação ao que estava planejado. O indicador construído para essa variável é: quantidade de ligações realizadas pelo Programa LPT no estado "X" dividido pela quantidade de ligações planejadas para 0 estado "X”. Nesse caso, houve a classificação do desempenho dos estados em quatro categorias, conforme o alcance das metas do Programa: 1) Desempenho baixo - 0\% a 50\%; 2) Desempenho regular - 50,01\% a $89,9 \%$; 3) Desempenho bom - 90\% a 120\%; e 4) Desempenho ótimo - acima de $120,01 \%$. Essa delimitação foi adotada tendo em vista que um desempenho acima de $90 \%$ na execução física de uma política pública é considerado satisfatório, conforme avaliações de Programas Governamentais realizadas pelo Ministério do Planejamento, Orçamento e Gestão.

A variável independente/antecedente "Novos atores" está relacionada aos novos atores que surgiram durante o processo de implementação do Programa LPT que não haviam sido contemplados ou participado do processo de elaboração dessa política pública, ou seja, são os atores que demonstraram interesse direto na época da execução do LPT e não tinham sido identificados anteriormente como atuantes nesse processo.

Os atores identificados no processo de formulação da política que participariam da implementação do programa são: 1) Ministério de Minas e Energia; 2) Centrais Elétricas Brasileiras S.A. - Eletrobras; 3) Estados; 4) Concessionárias e Permissionárias de Energia Elétrica-Agente Executor;5) Agência Nacional de Energia Elétrica - ANEEL. Logo, os demais atores que não fazem parte dessa lista, mas atuaram diretamente e influenciaram a condução dessa política pública, foram considerados novos atores.

Os indicadores para mensurar essa variável são: 1) Atuação de novos atores que contribuíram positivamente para a implementação do Programa LPT; e 2) Atuação de novos atores que dificultaram o processo de implementação do Programa LPT.

O primeiro indicador da variável independente "novos atores" - "Atuação de novos atores que contribuíram positivamente para a implementação do Programa LPT" - busca identificar se houve ou não participação positiva de novos atores no processo de implementação desse programa. Esse indicador pode ser considerado um fator relevante para analisar esse processo, pois a atuação de novos atores que contribuem para facilitar a implementação desse programa influencia diretamente e de forma positiva o desempenho dessa política pública. Logo, para efeito da análise desse indicador, os valores que ele poderia assumir são: $A$ ) (SIM $=1)$; ou $B$ ) (NÃO = 0), sendo que "SIM" quer 
dizer que houve atuações de novos atores que contribuíram para o processo de implementação do Programa LPT em determinado estado e "NÃO" significa que não houve. O mesmo tratamento foi dado ao indicador "Atuação de novos atores que contribuíram negativamente para a implementação do Programa LPT". Porém, cabe ressaltar que esse indicador busca identificar se houve ou não a participação de novos atores nesse programa que prejudicaram/ atrasaram sua execução.

A coleta de dados foi realizada em três etapas. $\mathrm{Na}$ primeira, foi feita a pesquisa documental. Em um segundo momento, foram efetuadas as entrevistas semiestruturadas com os gestores nacionais do Programa LPT para levantar informações sobre a atuação e a influência de novos atores no processo de implementação dessa política pública nos estados brasileiros. Foram realizadas sete entrevistas. ${ }^{2}$ Os entrevistados foram: o secretário de Energia Elétrica; o coordenador nacional do Programa LPT; os coordenadores das regiões geoelétricas Sul, Centro-Oeste/Sudeste, Nordeste e Norte, e um ex-coordenador das regiões geoelétricas Sul e Nordeste. Eles foram escolhidos para a realização da entrevista em razão de suas responsabilidades em relação à gestão do Programa LPT e ao conhecimento sobre o histórico do processo de implementação dessa política pública. Cabe ressaltar que, de acordo com Creswell (2010), as entrevistas são úteis quando os participantes não podem ser diretamente observados e têm a vantagem de possibilitar que o pesquisador busque informações históricas não documentadas (MARCONI; LAKATOS, 2010).

Por fim, a terceira etapa envolveu o encaminhamento do questionário aos participantes dos 26 Comitês Gestores Estaduais (CGE) do Programa LPT. Importa registrar que os CGE possuem a atribuição de discutir soluções para os problemas que surgem durante 0 processo de implementação do Programa LPT nos diferentes estados brasileiros, fato esse que justifica a importância de averiguar, no âmbito desses comitês, as opiniões de seus integrantes acerca do processo de implementação dessa política pública. Cabe destacar que os CGE são compostos por pessoas de diferentes organizações que atuam no processo de implementação do Programa LPT, sendo elas: representantes dos agentes executores, dos governos estaduais e da sociedade civil.

Foram recebidos 87 questionários respondidos pelos integrantes dos CGE parte dos dados quantitativos da pesquisa -, que foram analisados por meio do software estatístico Statistical Package for Social Science (SPSS), versão 18.0. O teste estatístico realizado foi o de frequência de ocorrência das respostas dos itens dos questionários. $^{3}$

As informações coletadas foram analisadas por meio do método Qualitative Comparative Analisys (QCA), utilizando o software de análise comparativa - Tosmana (CRONQVIST, 2007). Por meio desse método, obtêm-se equações, ou seja, combinações ou configurações lógicas das variáveis que apresentem as condições necessárias e/ou suficientes para alcançar um determinado resultado. No que se refere a essa pesquisa, são as condições necessárias e/ou suficientes, sob a ótica da variável "Novos atores" para alcançar os desempenhos "baixo", "regular", "bom" e "ótimo" do Programa Luz para Todos.

Cabe ressaltar que uma das premissas 
do método QCA é a equifinalidade, ou seja, pode haver mais de uma possibilidade ou combinações de variáveis que expliquem o alcance de um mesmo resultado. Além disso, esse método utiliza álgebra booleana para realizar a análise comparativa na base da presença ou ausência de condições que levem a determinado resultado da variável dependente. Sendo assim, o QCA reporta, por meio de formulações/equações lógicas, as configurações de condições necessárias e/ou suficientes para um dado resultado.

Dessa forma, a pesquisa utiliza técnicas da abordagem qualitativa para a realização do estudo do fenômeno da influência da atuação de novos atores no processo de implementação e como eles se associam à variação de desempenho observada no âmbito dessa política pública.

\section{Descrição dos casos}

Nesta seção, apresenta-se uma descrição sucinta do processo de implementação do Programa LPT nos diferentes estados brasileiros, sob a ótica da atuação de novos atores no processo de execução dessa política pública. Importa destacar que eles foram congregados por regiões geoelétricas - região geoelétrica Sul; região geoelétrica Centro-Oeste/Sudeste; região geoelétrica Norte; e região geoelétrica Nordeste -, tendo em vista que a gestão do programa ocorre em conformidade com esse agrupamento.

A região geoelétrica Sudeste/CentroOeste compreende os estados de Goiás, Rio de Janeiro, São Paulo, Espírito Santo e Minas Gerais. Nessa região, o estado de Goiás apresentou desempenho "regular" no processo de implementação do Programa LPT, enquanto os demais estados tiveram desempenho "ótimo". Conforme relato do coordenador da região geoelétrica CentroOeste/Sudeste do Programa LPT, no processo de execução do Programa LPT nos estados de Goiás, São Paulo, Espírito Santo e Minas Gerais, não houve atuação de nenhum novo ator que contribuiu positiva ou negativamente para a implementação dessa política pública, exceto no estado do Rio de Janeiro, que a atuação de órgãos ambientais foi um fator que pode ter prorrogado/postergado a execução do Programa LPT, tendo em vista a morosidade na concessão das licenças.

A região geoelétrica Sul abrange os estados de Mato Grosso do Sul, Paraná, Rio Grande do Sul e Santa Catarina. Nessa região, todos os estados apresentaram desempenho "ótimo" no processo de implementação do Programa LPT. De acordo com o relato do coordenador da região geoelétrica Sul do Programa LPT, no processo de sua execução nos estados do Mato Grosso do Sul e do Paraná, cabe destacar a participação das Prefeituras Municipais, da Eletrosul e do Instituto Nacional de Colonização e Reforma Agrária (Incra) como atores novos que contribuíram para facilitar a implementação do programa nesses estados. Além disso, nos estados de Santa Catarina e do Rio Grande do Sul, importa registrar, conforme entrevista realizada com o coordenador da região geoelétrica Sul, que a participação das Prefeituras Municipais e da Eletrosul foram extremamente relevantes, tendo em vista que também se destacaram como atores que contribuíram para facilitar a execução do Programa nessas regiões. Por fim, cabe ressaltar que, na região geoelétrica Sul, não foram identificados novos atores que poderiam ter dificultado a execução do Programa LPT nos estados que compõem essa região. 
A região geoelétrica Norte compreende os estados do Acre, Amazonas, Amapá, Maranhão, Mato Grosso, Pará, Rondônia, Roraima e Tocantins. Nessa região, os estados do Acre, Amazonas, Amapá, Roraima e Rondônia apresentaram desempenho "baixo" no processo de implementação do Programa LPT, os estados do Maranhão, Pará e Tocantins tiveram desempenho "regular" e, por fim, o estado do Mato Grosso obteve desempenho "ótimo". De acordo com o relato do coordenador da região geoelétrica Norte do Programa LPT, no processo de execução do Programa LPT nessa região, em todos os estados, houve atuação de órgãos ambientais, que possivelmente contribuíram para atrasar a execução dessa política pública em razão da morosidade na concessão de licenças ambientais para a realização das obras de eletrificação rural. Em relação à atuação de atores que poderiam ter contribuído para dar agilidade ao processo de implementação do Programa LPT nesses estados, o coordenador da região geoelétrica não ressaltou nenhuma situação em que esse fato tivesse ocorrido.

Por fim, a região geoelétrica Nordeste compreende os estados de Alagoas, Bahia, Ceará, Paraíba, Piauí, Pernambuco, Rio Grande do Norte e Sergipe. Nessa região, o estado do Piauí apresentou desempenho "baixo" no processo de implementação do Programa LPT, os estados da Bahia e da Paraíba tiveram desempenho "regular", os estados de Alagoas, Ceará e Pernambuco obtiveram desempenho "baixo" e, por último, os estados do Rio Grande do Norte e de Sergipe tiveram desempenho "ótimo".

Conforme relato do coordenador da região geoelétrica Nordeste do Programa LPT, no processo de execução do Programa LPT nessa região, em todos os estados, houve atuação de órgãos ambientais, que possivelmente contribuíram para atrasar a execução dessa política pública em decorrência à morosidade na concessão de licenças ambientais para a realização das obras de eletrificação rural. Por outro lado, houve também, em todos os estados, atuação de outros atores como o Incra e algumas Prefeituras Municipais, que contribuíram positivamente para a implementação do Programa LPT nessa região. Cabe ressaltar ainda que, no estado do Ceará, o Conselho de Consumidores de Energia (CCE) foi um ator relevante pelo apoio positivo dado à implementação do programa nesse estado. 
Tabela1 - Identificação de "novos atores" que atuaram no processo de implementação do Programa LPT e a relação do desempenho observado em cada estado.

\begin{tabular}{|c|c|c|c|c|}
\hline \multirow{2}{*}{$\begin{array}{c}\text { REGIÃO } \\
\text { GEOELÉTRICA }\end{array}$} & \multirow{2}{*}{ ESTADO } & \multicolumn{2}{|c|}{ NOVOS ATORES } & \multirow{2}{*}{ DESEMPENHO } \\
\hline & & POSITIVO & NEGATIVO & \\
\hline \multirow{5}{*}{ CENTRO-OESTE/SUDESTE } & GO & NAO & NÃO & REGULAR \\
\hline & MG & NAOO & NÃO & отімо \\
\hline & SP & NAOO & NÃO & о́тімо \\
\hline & ES & NÄO & NÃO & оттмо \\
\hline & RJ & NÅO & $\begin{array}{c}\text { SIM } \\
\text { (ÓRGÃOS AMBIENTAIS) }\end{array}$ & оттмо \\
\hline \multirow{4}{*}{ SUL } & MS & $\begin{array}{c}\text { SIM } \\
\text { (PREFEITURAS MUNICIPAIS; } \\
\text { ELETROSUL; INCRA) }\end{array}$ & NAO & оттмо \\
\hline & PR & $\begin{array}{c}\text { SIM } \\
\text { (PREFEITURAS MUNICIPAIS; } \\
\text { ELETROSUL; INCRA) }\end{array}$ & NÃO & оттмо \\
\hline & RS & $\begin{array}{c}\text { SIM } \\
\text { (PREFEITURAS MUNICIPAIS: } \\
\text { ELETROSUL) }\end{array}$ & NÃO & о́тімо \\
\hline & SC & $\begin{array}{c}\text { SIM } \\
\text { (PREFEITURAS MUNICIPAIS: } \\
\text { ELETROSUL) }\end{array}$ & NÃO & оттмо \\
\hline \multirow{9}{*}{ NORTE } & $A C$ & NAOO & $\begin{array}{c}\text { SIM } \\
\text { (ÓRGÃOS AMBIENTAIS) }\end{array}$ & BAIXO \\
\hline & AM & NÅO & $\begin{array}{c}\text { SIM } \\
\text { (ÓRGÃOS AMBIENTAIS) }\end{array}$ & BAIXO \\
\hline & AP & NÅO & $\begin{array}{c}\text { SIM } \\
\text { (ÓRGAOS AMBIENTAIS) }\end{array}$ & BAIXO \\
\hline & RO & NÅO & SIM & BAIXO \\
\hline & RR & NÅOO & $\begin{array}{c}\text { SIM } \\
\text { (ÓRGÅOS AMBIENTAIS) }\end{array}$ & BAIXO \\
\hline & MA & NÅOO & $\begin{array}{c}\text { SIM } \\
\text { (ÓRGÃOS AMBIENTAIS) }\end{array}$ & REGULAR \\
\hline & PA & NÅOO & $\begin{array}{c}\text { SIM } \\
\text { (ÓRGAOOS AMBIENTAIS) }\end{array}$ & REGULAR \\
\hline & Tо & NĂO & (ÓRGÃOS AMBIENTAIS) & REGULAR \\
\hline & MT & NÅO & $\begin{array}{c}\text { SIM } \\
\text { (ÓRGÄOS AMBIENTAIS) }\end{array}$ & оттмо \\
\hline \multirow{8}{*}{ NORDESTE } & PI & $\begin{array}{c}\text { SIM } \\
\text { (PREFEITURAS MUNICIPAIS; INCRA) }\end{array}$ & $\begin{array}{c}\text { SIM } \\
\text { (ORGÄOS AMBIENTAIS) }\end{array}$ & BAIXO \\
\hline & BA & $\begin{array}{l}\text { SIM } \\
\text { (PREFEITURAS MUNICIPAIS; INCRA) }\end{array}$ & SIM & REGULAR \\
\hline & PB & $\begin{array}{c}\text { SIM } \\
\text { (PREFEITURAS MUNICIPAIS; INCRA) }\end{array}$ & $\begin{array}{c}\text { SIM } \\
\text { (ÓRGÄOS AMBIENTAIS) }\end{array}$ & REGULAR \\
\hline & AL & $\begin{array}{l}\text { SIM } \\
\text { (PREFEITURAS MUNICIPAIS; INCRA) }\end{array}$ & SIM & вом \\
\hline & $\mathrm{CE}$ & $\begin{array}{c}\text { SIM } \\
\text { (PREFEITURAS MUNICIPAIS; INCRA) }\end{array}$ & $\begin{array}{c}\text { SIM } \\
\text { (ÓRGÃOS AMBIENTAIS) }\end{array}$ & вом \\
\hline & PE & $\begin{array}{c}\text { SIM } \\
\text { (PREFEITURAS MUNICIPAIS; INCRA) }\end{array}$ & $\begin{array}{c}\text { SIM } \\
\text { (ÓRGAOOS AMBIENTAIS) }\end{array}$ & вом \\
\hline & $\mathrm{RN}$ & $\begin{array}{c}\text { SIM } \\
\text { (PREFEITURAS MUNICIPAIS; INCRA) }\end{array}$ & $\begin{array}{c}\text { SIM } \\
\text { (ÓRGÃOS AMBIENTAIS) }\end{array}$ & оттмо \\
\hline & SE & $\begin{array}{l}\text { SIM } \\
\text { (PREFEITURAS MUNICIPAIS: } \\
\text { INCRA; CCE) }\end{array}$ & $\begin{array}{c}\text { SIM } \\
\text { (ORGAOS AMBIENTAIS) }\end{array}$ & оттмо \\
\hline
\end{tabular}

Fonte: elaborado pelo autor. 


\section{Análise e resultados}

A análise foi realizada por meio do método QCA para demonstrar como a variável "Novos atores" influencia o desempenho do Programa LPT, levando em consideração que ela é composta por dois indicadores: 1) Atuação de novos atores que contribuíram positivamente para a implementação do Programa LPT, e 2) Atuação de novos atores que dificultaram o processo de implementação do Programa LPT. Foram identificadas situações em que indicadores da variável "Novos atores" contribuíram como condições necessárias e suficientes para alcançar os resultados "baixo", "regular" ou "bom" relacionados ao desempenho do Programa LPT.

No caso da relação entre a variável "Novos atores" e o desempenho "ótimo", no entanto, chegou-se à equação lógica para expressar as condições que resultam nesse desempenho. Conforme 0 resultado da análise, a configuração para o alcance do desempenho "ótimo" é composta pela presença de novos atores que atuaram positivamente no processo de implementação do Programa LPT e a ausência de novos atores que dificultaram a implementação dessa política pública.

Os casos que apresentaram essa configuração são os estados do Paraná, Santa Catarina, Rio Grande do Sul e Mato Grosso do Sul, localizados na região geoelétrica Sul. Importa ressaltar que, quando o coordenador da região geoelétrica Sul foi questionado sobre a participação de novos atores que contribuíram negativa ou positivamente para a implementação do Programa LPT, ele se manifestou da seguinte forma:

Houve outros atores participantes que entraram no decorrer na implementação do programa, que viram no programa, talvez, uma solução para vários problemas. Como exemplo, eu cito o Incra (Coordenador da região geoelétrica Sul).

Quando o coordenador da região geoelétrica Sul foi instado a dizer em qual estado especificamente houve a participação do Incra, ele destacou: "Certamente em todos eles [da região geoelétrica Sul]. Em todos os estados houve a participação do Incra". E, por fim, quando foi perguntado especificamente se houve algum novo ator que prejudicou, de forma significativa, a implementação do Programa, o mesmo coordenador respondeu:

Não. Houve aqueles processos normais, vamos dizer assim, de fiscalização dos órgãos ambientais. E o que houve na verdade foi a colaboração de alguns órgãos a nível municipal e estadual no sentido de liberar licenciamento de obras, enfim... porque na verdade esses entraves, houve aqueles entraves normais, vamos dizer assim, de demora, de análise de projetos... que muitas vezes os estados com seus órgãos estaduais tentaram resolver, solucionar. Agilizar, vamos dizer assim (Coordenador da região geoelétrica Sul).

Por sua parte, o coordenador da região geoelétrica Norte caracteriza como negativa a atuação de órgãos de controle ambiental, conforme exposição a seguir, fato esse que prejudicou a implementação do Programa LPT em algumas regiões:

Teve na região Norte de uma forma geral, os órgãos ambientais, eles contribuíram negativamente. Porque houve muitas exigências que não estavam muito bem 
definidas. Em relação, de uma forma geral, às redes de distribuição, elas já são definidas como um trabalho que não gera impacto ambiental por si só. A partir das redes de transmissão é que tem o impacto ambiental significativo. Mas muitos órgãos de controle passaram a considerar as redes de distribuição... órgãos de controle ambiental, passaram a considerar a rede de distribuição como impactante também e aí começaram a exigir licenças, vários tipos de licenças e isso atrasou bastante algumas regiões (Coordenador da região geoelétrica Norte).

A importância dessa variável é ratificada pelo coordenador nacional do Programa LPT ao qualificar a atuação de alguns órgãos de fiscalização e controle no processo de implementação do Programa LPT, conforme exposto a seguir:

Eu acho que a gente pode separar em duas situações. Você tem os órgãos de controle que tem a função de fiscalizar e cuidar para que a aplicação do recurso público seja feita de forma legal, transparente e racional... que ele nos ajuda, esses órgãos nos ajudam à medida que eles nos chamam a atenção para as melhorias de controle para melhor aplicação desses recursos. Então, contribuíram muito. Eu não julgo que os órgãos de controle tenham dificultando muito, pelo contrário, ajudaram muito a implementar, inclusive, alguns procedimentos de melhoria de gestão do Programa. E você tem um outro conjunto de órgãos que são órgãos licenciadores que aí tem a função de licenciar as obras e cuidar para que as obras atendam os requisitos legais, sejam eles de proteção ambiental, seja eles aí de outra ordem... que eu diria que eles dificultam na medida que eles são morosos, mais morosos nos procedimentos, não que eles não deixem de ser exigentes e que as empresas não tenham que cumprir as exigências aí para execução das obras. Mas eu penso que alguns, em algumas situações, acabaram dilatando os cronogramas em razão da morosidade desses licenciamentos ou das decisões (Coordenador nacional do Programa LPT).

O fato de o desempenho "ótimo" ser caracterizado pela ausência de novos atores que prejudicassem a implementação do Programa LPT e pela presença de novos atores que facilitassem a execução dessa política pública também foi corroborado pelo resultado dos questionários respondidos pelos integrantes dos CGE, daqueles estados que alcançaram o desempenho "ótimo". A maioria discorda totalmente que novos atores prejudicaram a implementação do Programa LPT e concorda que novas organizações que participaram do processo de implementação do programa foram fundamentais para seu sucesso e contribuíram positivamente para o alcance dos resultados planejados.

Assim sendo, esse é o conjunto de explicações que fundamenta a relação da variável "Novos atores" com os desempenhos do Programa LPT, demonstrada pela utilização do software Tosmana.

Apesar de a análise não ter identificado condições necessárias e suficientes para o alcance dos desempenhos "ruim", "regular" e "bom", o resultado dos questionários dá indicativos de que há relação entre as variáveis "Desempenho do Programa LPT" e "Novos atores". Em relação aos estados que alcançaram os desempenhos "ruim" e "regular", a maioria dos integrantes dos CGE 
desses estados concorda que a atuação de órgãos ambientais dificultou a implementação do Programa LPT e discorda totalmente que novos atores tenham sido fundamentais para o sucesso da implementação dessa política pública. Por fim, em relação aos estados que alcançaram o desempenho "bom", a maioria dos representantes dos CGE desses estados discorda totalmente que a participação de novos atores prejudicaram ou facilitaram o processo de implementação do Programa LPT nessas regiões.

Sendo assim, quanto à relação dos resultados alcançados, importa registrar diferenças significativas nos resultados apontados na literatura acadêmica sobre a implementação de políticas públicas. Pressman e Wildavsky (1984) e Lundin (2007) destacam que o processo de execução de uma política pública apresenta problemas em razão de o número de atores envolvidos nesse processo ser excessivo, e Matland (1995) aponta que os teóricos da vertente top-down argumentam que, para minimizar os problemas de uma política pública, deve-se reduzir a quantidade de atores envolvidos no processo de implementação.

Diferentemente disso, no caso do Programa LPT, foi evidenciado que, para o alcance do desempenho "ótimo", os fatores condicionantes são a existência de novos atores que apoiaram a implementação do Programa LPT e a ausência de novos atores que prejudicaram/ atrasaram a execução dessa política pública. Portanto, a qualidade da participação também se apresenta como relevante, fato não mencionado em estudos anteriores.

Nessa situação, especificamente do Programa LPT, o Incra, por exemplo, foi identificado como novo ator com interesse direto no processo de implementação dessa política pública, tendo em vista que os novos assentamentos rurais seriam contemplados pelo acesso à energia elétrica à medida que o Programa progredisse. As prefeituras municipais, citadas na pesquisa, atuaram de forma a dar rapidez à concessão das licenças de obras, enquanto a Eletrosul ofereceu logística e infraestrutura local para a implementação do Programa LPT, buscando agilizar o provimento de energia elétrica a diversos domicílios localizados na zona rural, em especial, aqueles que apresentavam dificuldade de acesso. Os Conselhos de Consumidores de Energia, por sua vez, auxiliaram na identificação de novos beneficiários do programa, proporcionando maior agilidade no atendimento ao públicoalvo dessa política pública. Sendo assim, os interesses desses novos atores estavam fortemente alinhados e articulados às diretrizes de execução do Programa LPT, atuando de forma a contribuir positivamente para a implementação dessa política.

Por outro lado, os interesses dos órgãos de controle ambiental não estavam completamente alinhados às diretrizes de universalização do acesso à energia no meio rural, tendo em vista que as obras de eletrificação rural poderiam gerar impactos ambientais. Dessa forma, essas organizações foram identificadas como novos atores que proporcionaram atrasos na realização das atividades do Programa LPT em razão da morosidade na análise das informações para a concessão das licenças ambientais, necessárias para dar andamento à execução dessa política pública.

\section{Considerações finais}

propósito deste artigo foi descrever a 
influência de novos atores no processo de implementação do Programa LPT e analisar como ela se associa à variação de desempenho observada no âmbito dessa política pública. Os resultados sugerem que a quantidade de novos atores envolvidos no processo de implementação de uma política pública não é condição necessária e/ou suficiente para caracterizar o sucesso ou o insucesso da execução dessa política pública, contrário ao que aponta a literatura de implementação (PRESSMAN; WILDAVSKY, 1984; LUNDIN, 2007). As evidências do estudo apontam fortemente à conclusão de que não é necessariamente a quantidade de atores que definirá se uma determinada política pública obterá sucesso ou não, mas sim sua contribuição para o processo de implementação, ou seja, a qualidade da participação.

A partir das análises realizadas pelo método QCA, foram identificadas duas condições necessárias para o alcance de desempenho "ótimo" no processo de implementação do Programa LPT: ausência de novos atores que dificultam a implementação do Programa LPT e presença de novos atores que facilitam a execução dessa política pública.

Nessesentido, importa registrarcontribuições significativas do estudo realizado para a literatura acadêmica de implementação de políticas públicas. De acordo com a literatura desse tema, quanto maior a quantidade de atores envolvidos no processo de execução de uma política pública, maiores serão as dificuldades do processo de implementação (PRESSMAN; WILDAVSKY, 1984; LUNDIN, 2007). No entanto, os resultados alcançados com essa pesquisa demonstram que a quantidade de atores no processo de implementação de políticas públicas não é suficiente para caracterizar o desempenho do processo de execução, nem o alcance do sucesso ou insucesso no processo de implementação de uma política. A qualidade dessa participação também é importante.

Foi evidenciado que novos atores tanto podem facilitar quanto prejudicar 0 processo de implementação. Há, portanto, a necessidade de qualificar a participação desses atores, classificando-os em atores que facilitam a execução da política e atores que dificultam sua execução. Dessa forma, o resultado dessa pesquisa contribuiu para aprimorar o entendimento da atuação dos atores no processo de implementação de políticas públicas, associando-os não mais à quantidade, mas à forma de atuação no processo de implementação, ou seja, a qualidade de sua atuação.

Tendo em vista o exposto, conclui-se que a variável "Novos atores" foi importante para compreender o processo de implementação do Programa LPT nos diferentes estados brasileiros, bem como distinguir os fatores condicionantes para oalcance do desempenho "ótimo", observado no âmbito dessa política pública. No entanto, a importância desta variável parece não depender da quantidade de novos atores, mas da qualidade de sua participação.

Pode-se concluir também que o método utilizado para identificar, descrever e caracterizar a influência de novos atores no processo de implementação do Programa LPT e analisar como ela está associada à variação de desempenho observada no âmbito dessa política pública foi muito relevante para o alcance dos objetivos propostos. $O$ método QCA foi capaz de proporcionar as ferramentas necessárias para a realização 
deste estudo. A escolha do método qualitativo também demonstrou ser relevante para a análise da complexidade do processo de implementação do Programa LPT. Além disso, o desenho da pesquisa utilizou várias técnicas de triangulação, que contribuíram para dar robustez às conclusões da pesquisa.

Não quer dizer que não há limitações ao estudo realizado. Uma delas diz respeito ao recorte do processo de implementação do Programa LPT. Embora o programa ainda está em fase de implementação, com previsão de término em 2014, a pesquisa realizada focalizou apenas a primeira etapa, o período de 2004 a 2008. Outra limitação ao que foi reportado aqui se refere ao fato de que, neste estudo, se tratou apenas da contribuição da variável "Novos atores" ao desempenho alcançado pelo Programa LPT, sendo que há outras identificadas pela literatura de implementação, que são relevantes a esse processo e que podem afetar o desempenho da execução. Além disso, essa pesquisa trouxe contribuições apenas para os casos de desempenho "ótimo" na implementação de uma única política pública e não proporcionou evidências que permitem compreender os casos de menor desempenho no programa estudado, não sendo possível generalizar e abranger outras políticas.

Mesmo assim, é possível, com base nos resultados da pesquisa, tecer algumas recomendações de natureza prática em relação à gestão do Programa LPT. Sugere-se que os coordenadores do programa façam uma análise prévia das condições que caracterizam o desempenho "ótimo" da implementação dessa política pública em determinado estado. Caso sejam identificados atores que possam prejudicar o andamento das obras de eletrificação rural, por exemplo, é importante buscar maneiras para mitigar o risco inerente à atuação desses atores por meio da articulação de interesses e coordenação de ações. Por outro lado, sugere-se que sejam identificadas instituições que se beneficiem dessa política pública e que possam contribuir para sua implementação.

Em termos gerais, essa pesquisa traz um novo olhar ao papel de novos atores no processo de implementação de uma política pública, focando não na quantidade de atores, como destaca a literatura de implementação, mas na qualidade da atuação, ou seja, na contribuição desses novos atores ao processo de implementação de uma dada política pública.

Para finalizar, propõe-se que novos estudos sejam realizados, no âmbito de outras políticas públicas na área de infraestrutura para averiguar se a configuração da atuação e influência de novos atores que caracterizaram o alcance do desempenho "ótimo" no processo de implementação do Programa LPT também seja similar à de outras políticas públicas dessa natureza. Além disso, no âmbito do próprio Programa, sugerem-se novos estudos, adicionando mais variáveis e contemplando todo o período de implementação dessa política pública.

\section{Referências}

BRASIL. Agência Nacional de Energia Elétrica. Resolução Normativa n. 175, de 28 de novembro de 2005. Brasília: ANEEL, 2005.

Agência Nacional de Energia Elétrica. Resolução Normativa n. 365, de 19 de maio de 2009. Brasília: ANEEL, 2009. 
Ministério de Minas e Energia.

Manual de Operacionalização do Programa Nacional de Universalização do Acesso e Uso da Energia Elétrica (Programa Luz para Todos), anexo à Portaria n. 85, de 20 de fevereiro de 2009.

Ministério de Minas e Energia. Pesquisa Quantitativa Domiciliar de Avaliação da Satisfação e de Impacto do Programa Luz para Todos. 2009.

- Ministério de Minas e Energia.

Luz para Todos: Um marco histórico - 10 milhões de brasileiros saíram da escuridão. 2010.

Decreto n. 4.873, de 11 de novembro de 2003.

Decreto n. 6442, de 25 de abril de 2008.

Decreto n. 7.324, 5 de outubro de 2010.

CRESWELL, J. W. Projeto de pesquisa: método qualitativo, quantitativo e misto. Porto Alegre: Artmed, 2010.

CRONQVIST, LASSE. TOSMANA TOOI for Small-N Analysis. Version 1.3 beta. User Manual. [S.I.]: Lasse Cronqvist, 2007. Disponível em: <www.tosmana.net>. Acesso em: 20 jul. 2012.

GEORGE, A. L.; BENNET, A. Case studies and theory development in social sciences. Cambridge: MIT Press, 2005.

HOWLETT, M; RAMESH, M. Studying Public Policy: Policy Cycles and Policy
Subsystems. Oxford University Press. 1995.

HANF, K.; TOONEN, T. A. J. Policy implementation in federal and unitary systems: questions of analysis and design. Rotterdam: Martinus Nijhoff Publishers, 1985.

HILL, M.; HUPE, P. Implementing public policy: governance in theory and in practice. London: Sage Publications, 2005.

LOWI, T. Four Systems of Policy, Politics, and Choice. Public Administration Review, 32:298-310, 1972.

LUNDIN, Martin. When does cooperation improve public policy implementation? The Policy Studies Journal, v. 35, n. 4, 2007.

MATLAND, R. E. Synthesizing the implementation literature: the ambiguityconflict model of policy implementation. Houston: Journal of Public Administration, v. 5, n. 2. abr., p. 145-174, 1995.

MAZMANIAN, D. A.; SABATIER, P. A. Implementation and Public Policy with a New Postscript. University Press of America. 1989.

MARCONI, M. A.; LAKATOS, E. M. Metodologia científica. Editora Atlas S.A., 5. ed., 2010.

O'TOOLE, L. Jr. Research on Policy Implementation: Assessment and Prospects. Journal of Public Administration Research and Theory, p.263-288, 2000.

Rational choice and the public management of interorganizational networks. In: KETTL, D. F.; MILWARD, H. B. Public management. Baltimore: The Johns Hopkins 
University Press, 1996.

MONTJOY, R. S. Interorganizational Policy Implementation: A Theoretical Perspective. Public Administration Review. v. 44, n. 6, p. 491-503 (nov./dec., 1984).

PETTIGREW, A. M. (1990). Longitudinal Field Research on Change: Theory and Practice. Organization Science, n. 1, v. 3, 1990.

PIERSON, P. Public policies as institutions. In: SHAPIRO, I.; SKOWRONEK, S.; GALVIN, D. (eds.). Rethinking political institutions: the art of the state. New York University Press, 2006.

PRESSMAN, J. I.; WILDAVSKY, A. Implementation: how great expectations in Washington are dashed in Oakland: or, Why it's amazing that Federal programs work at all, this being a saga of the Economic Development Administration as told by two sympathetic observers who seek to build morals on a foundation of ruined hopes. University of California Press, 3rd edition. Berkeley, Los Angeles, London. 1984.

RAGIN, C. C. The comparative method. Berkley: University of California Press, 1987.

Fuzzy-set social science. Chicago: The University of Chicago Press, 2000.

RIPLEY, R. Stages of policy process. In: MCCOOL, D. C. Public policy theories, models, and concepts: an anthology. New Jersey: Prentice Hall, 1995.

RUA, M. G. Análise de Políticas Públicas: conceitos básicos. In: RUA, M. G; CARVALHO, M. I. V. (Orgs.). Análise de Políticas Públicas: conceitos básicos. Brasília: Paralelo 15, 1998.
SCHWARTZ-SHEA, P. Judging Quality: Evaluative Criteria and Epistemic Communities. In: Yanow, D.; SchwartzShea, P. (Eds.). Interpretation and method: empirical research methods and the interpretive turn. New York: M. E. Sharpe, p. 82-113.

SILVA, P. L. B. e MELO, M. A. B. O processo de implementação de políticas públicas no Brasil: características e determinantes da avaliação de programas e projetos. Campinas: NEPP, Unicamp, Caderno n. 48, 2000.

SUBIRATS, J. Analisis de politicas publicas y eficacia de la administracion. Ministerio para las Administraciones Publicas. Colección Estudios: Madrid. 1992.

THEODOULOU, S. Z. Policy and Politics in Six Nations: A Comparative Perspective on Policy Making. Prentice-Hall, 2002.

\section{Notas}

1. Cabe destacar que este estudo é parte de um projeto maior, no qual foram identificadas diversas variáveis que poderiam influenciar o processo de implementação do Programa LPT.

2. As questões relacionadas à variável "Novos atores" nas entrevistas realizadas para essa pesquisa foram: 1) Quais foram os principais fatores que contribuíram para a implementação do Programa LPT nos Estados? Descrever por Estado. 2) Quais foram os principais fatores que prejudicaram a implementação do Programa LPT nos Estados? Descrever por Estado. 3) Houve - aparecimento de algum novo ator, organização pública, privada ou terceiro 
setor que não participou na fase de formulação do programa/projeto no Estado? Houve impacto desse novo ator no processo de implementação do Programa? Que tipo de impacto? Descrever por Estado.

3. As questões relacionadas à variável "Novos atores" do questionário elaborado para a realização dessa pesquisa foram: 1) Houve participação de novas organizações no processo de implementação do Programa LPT, que não sejam o MME, a Eletrobras, o Governo do Estado, a ANEEL ou o Agente Executor? 2) A implementação do Programa foi prejudicada por alguma outra organização que não seja o MME, a Eletrobras, o Governo do Estado, a ANEEL ou o Agente Executor? 3) Houve dificuldade de implementação do
Programa devido à demanda de órgãos de controle (CGU ou TCU, por exemplo)? 4) Houve dificuldade de implementação do Programa devido à demanda de órgãos do meio ambiente? 5) Outras organizações, que não sejam o MME, a Eletrobras, o Governo do Estado, a ANEEL ou o Agente Executor foram fundamentais para o sucesso na implementação do Programa? 6) A participação dessas novas organizações no processo de implementação contribuiu positivamente ao processo de implementação? As respostas dos questionários variavam de 1 (um) a 5 (cinco), sendo: 1 - Discordo totalmente; 2 - Discordo parcialmente; 3 - Concordo parcialmente; 4 - Concordo, e 5 - Concordo totalmente. 
ANEXO I - DESEMPENHO DO PROGRAMA LPT (2004-2008)

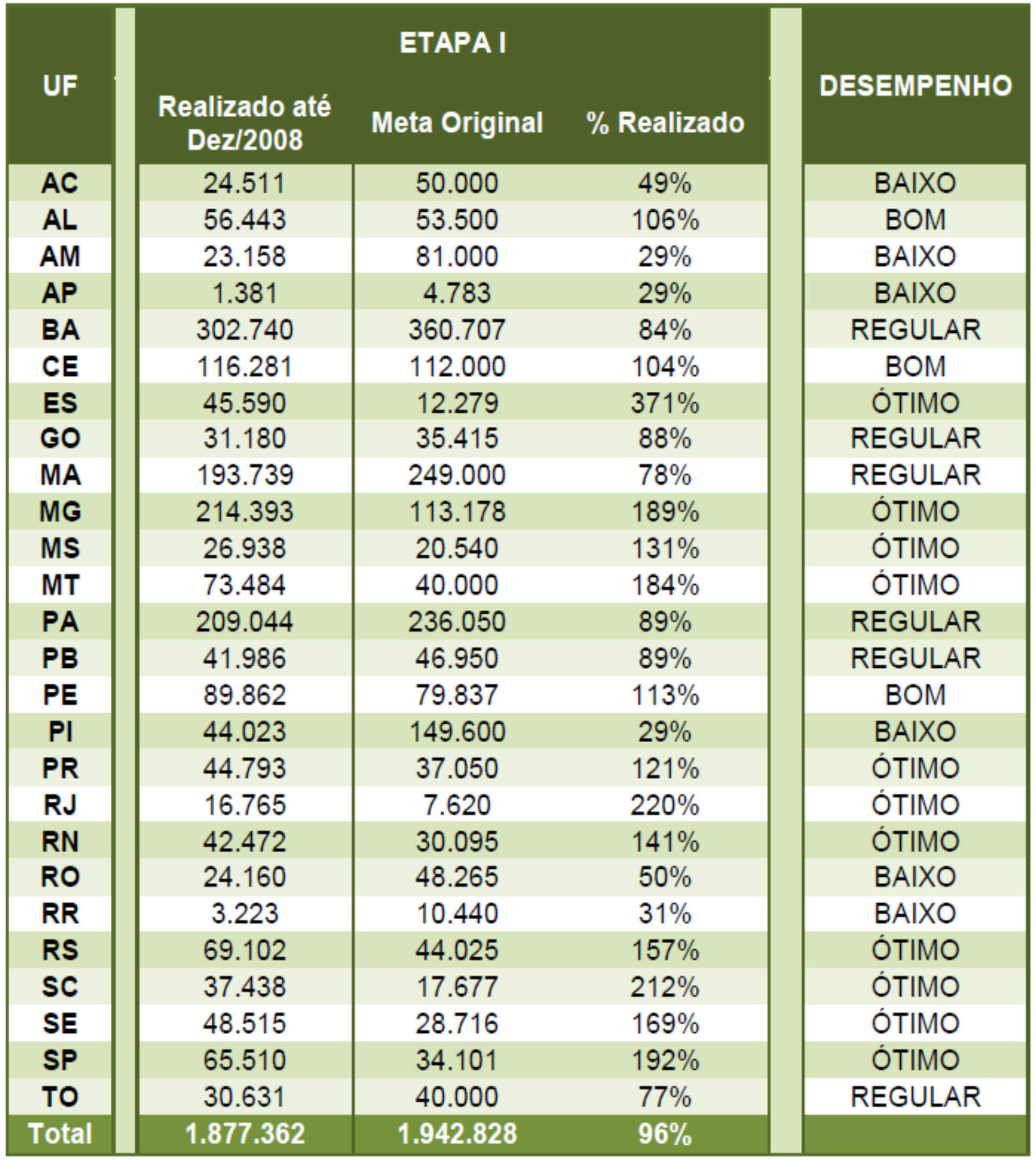

Fonte: Elaborado pelo autor. 